\title{
Immunoexpression of PD-L1 and PD-1 and Its Clinicopathological Correlation in Urothelial Carcinomas
}

\author{
Utpal Kumar ${ }^{1}$ Michael Leonard Anthony ${ }^{1}$ Rishabh Sahai ${ }^{1} \quad$ Ankur Mittal ${ }^{2}$ Prashant Durgapal ${ }^{1}$ \\ Sanjeev Kishore ${ }^{1}$ \\ ${ }^{1}$ Department of Pathology and Laboratory Medicine, AlIMS, \\ Rishikesh, Uttarakhand, India \\ 2 Department of Urology, AlIMS, Rishikesh, Uttarakhand, India \\ Address for correspondence Prashant Durgapal, MBBS, MD \\ Department of Pathology and Laboratory Medicine, AlIMS, \\ Rishikesh, 249203, Uttarakhand, India \\ (e-mail: prashant.path@aiimsrishikesh.edu.in).
}

J Lab Physicians 2022;14:197-201.

\begin{abstract}
Keywords

- immune checkpoint inhibitors

- PD-1

- PD-L1

- urothelial carcinoma

Introduction Urothelial carcinomas are the most common types of bladder tumors that have recently shown a changing trend in treatment protocols with the introduction and approval of immune checkpoint inhibitors. The most important immune checkpoint lies with the PD-1-PD-L1 axis. Although multiple drugs have been approved, there is uncertainty about patient selection criteria and diagnostic assays. Recent studies related to the laboratory-developed tests have opened up the horizon of PD-1 and PD-L1 immunohistochemistry even at resource-constrained laboratories. We propose to study these immunohistochemistry markers in our laboratory using newer clones.

Materials and Methods We selected 116 consecutive cases of transurethral bladder tumor resection from our laboratory archive and applied PD-1 and PD-L1 immunohistochemistry. The study was approved by the institution's ethics committee.

Results We found high expression of PD-1 and PD-L1 in urothelial carcinoma even with different cut-offs of PD-L1. Muscle invasion, lamina invasion, and grade of carcinoma had a statistically significant effect on the expression; however, age and sex did not affect the expression.

Conclusion Based on our current study, we can conclude that the clones used in our study show high expression in urothelial carcinoma and can aid in patient selection and treatment protocol, irrespective of age and sex.
\end{abstract}

\section{Introduction}

Programmed cell death protein 1 (PD-1 or CD 279) and its ligand programmed cell death ligand 1(PD-L1 or CD 274 or B7-H1) are cell-surface transmembrane molecules that play an important role in downregulating the immune system. Cancer cells express PD-L1 to avoid immunorecognition and destruction by imitating the signaling process of healthy cells. Currently, multiple immune checkpoint inhibitors including anti-PD-1 and anti-PD-L1 have been approved in published online November 2, 2021
DOI https://doi.org/

$10.1055 / \mathrm{s}-0041-1736480$. ISSN 0974-2727. (c) 2021. The Indian Association of Laboratory Physicians. All rights reserved.

This is an open access article published by Thieme under the terms of the Creative Commons Attribution-NonDerivative-NonCommercial-License, permitting copying and reproduction so long as the original work is given appropriate credit. Contents may not be used for commercial purposes, or adapted, remixed, transformed or built upon. (https://creativecommons.org/ licenses/by-nc-nd/4.0/)

Thieme Medical and Scientific Publishers Pvt. Ltd., A-12, 2nd Floor, Sector 2, Noida-201301 UP, India 

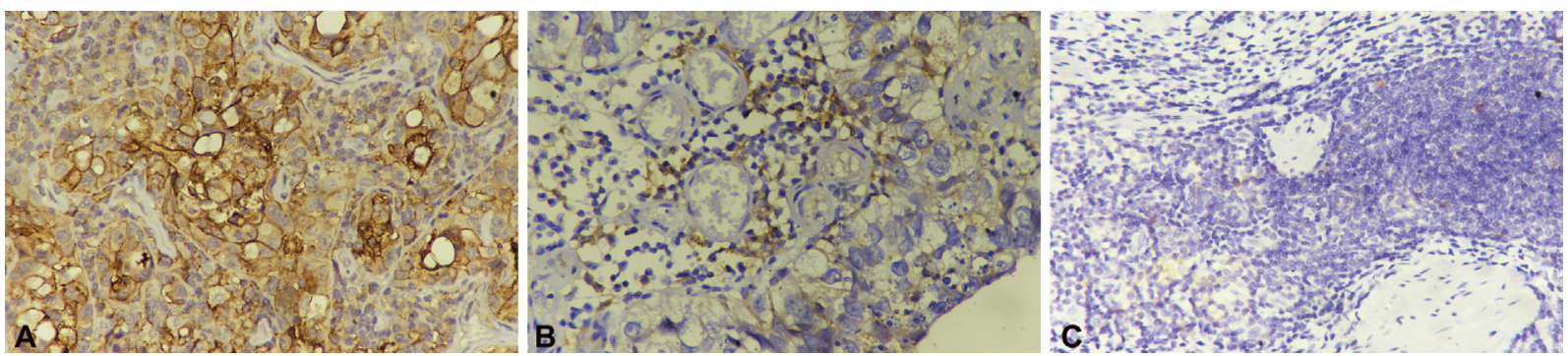

Fig. 1 (A) Strong PD-L1 positivity in tumor cells $(400 \times)$. (B) Strong PD-L1 positivity in lymphocytes along with weak positivity in tumor cells $(400 \times)$. (C) PD-1 positivity in lymphocytes $(400 \times)$.

metastatic and advanced urothelial carcinoma expanding the scope of treatment of urothelial carcinoma. Identifying patients who may or may not respond to PD1/PDL1 inhibitors is important as the majority of patients in different clinical trials did not have an overall response. ${ }^{1}$ Immunohistochemistry is the most commonly used assay in different clinical trials for patient selection. Although five immune checkpoint inhibitors have been approved in urothelial carcinoma, only two companion diagnostic immunohistochemistry assay has been approved by the US FDA. ${ }^{2}$ The Canadian Association of Pathologists-Association Canadienne Des Pathologistes (CAP-ACP) has recently published a guideline for Fit-ForPurpose PD-L1 biomarker testing for patient selection. ${ }^{3}$ This has opened up the space for validating new clones for PD-1 and PD-L1 immunohistochemistry assays. We tried to examine the expression of PD-L1 and PD-1 in our laboratory using new clones that have not yet been validated for patient selection for treatment in urothelial carcinoma.

\section{Methodology}

This study was a retrospective study and 116 consecutive cases of urothelial carcinoma received as TURBT (transurethral resection of urinary bladder tumors) chips were taken from the archived tissue blocks after the removal of duplicate cases. The cases included in the study did not receive any chemotherapy before surgery. Histopathological and immunohistochemical analyses were performed by two histopathology consultants independently. The examiners were blinded to histopathological diagnosis while interpreting immunohistochemistry slides. Clinical characteristics such as age, gender, and, smoking history were obtained from hospital records.

PD-L1 and PD-1 immunostaining were performed manually using prediluted antibodies for PD-1(clone NAT105, isotype mouse IgG, Biocare Medical, USA) and PD-L1 (clone CAL10, isotype Rabbit IgG, Biocare Medical, USA). Fourmicron-thick paraffin sections were brought to water. Heat-induced epitope retrieval method under steam pressure was used for antigen retrieval with $1 \%$ EDTA ( $\mathrm{pH} \mathrm{8.0)}$ as the retrieval buffer. This was followed by peroxidase blocking using Biocare's Peroxidase blocking system for 5 minutes and washing the sections twice. The slides were flooded with a primary antibody and incubated in dark at room temperature for 1 hour. After washing, slides were treated with horseradish peroxidase (HRP) for 10 minutes, followed by detection using the DAB detection system as chromogen and counterstained with hematoxylin. Known positive and negative controls were used with each batch of IHC staining.

Immunohistochemical staining was then assessed for PDL1 and PD-1. Membranous positivity in tumor cells and any cytoplasmic/membranous staining in mononuclear cells was considered as positive for PD-L1 ( - Fig. 1A, B). PD-L1 immunohistochemistry was scored as the percentage of positive cells. The cases were analyzed using a cut-off of both $1 \%$ and $5 \%$ for both lymphocytes and tumor cells separately. PD-1 was evaluated in mononuclear cells and any cytoplasmic/membranous positivity was taken as positive (-Fig. 1C). The staining percentage was calculated on the slides examined for the immunohistochemistry by calculating the percentage of lymphocytes or tumor cells showing staining.

The complete data were divided based on clinical and pathological features including age $(0-40,40-60,>60 \mathrm{yrs})$, smoking history (present, absent), gender (male, female), grade (high, low), lamina invasion (invasive, non-invasive), and muscle invasion (present, absent) (-Table $\mathbf{1}$ ).

$\mathrm{R}$ version 3.5.3 was used for statistical analysis. Fisher's exact test was used for comparison between different groups.

\section{Results and Interpretation}

Our study consisted of 116 patients. The clinicopathological characteristics of the study population are shown in - Table 1. Deep muscle biopsy could not be evaluated in 10 patients due to either the absence or marked crushing of deep muscle tissue. Smoking history was known in 90 cases only, which were used for analysis.

PD-1 expression was noted in $32.7 \%$ (38 of 116) of cases. The expression was seen in $43 \%$ ( 33 of 77) of high-grade cases and $49 \%$ ( 21 of 43 ) of muscle-invasive cases ( - Table 1 ).

PD-L1 expression in tumor cells was seen in $62 \%$ (72 of 116 ) and $50.8 \%$ (59 of 116) of cases, respectively, when the cut-offs of $1 \%$ and $5 \%$ were taken as positivity, respectively. PD-L1 expression in tumor-infiltrating lymphocytes was seen in 79.3\% (92 of 116) and 68.1\% (79 of 116) of cases, respectively, when the cut-offs of $1 \%$ and $5 \%$ were taken for positivity ( - Table $\mathbf{1}$ ).

Immunoexpression of PD-1 and PD-L1 showed no statistical difference in different age groups, gender, or smokers. PD-1 showed a higher expression in high-grade tumors 
Table 1 Clinicopathological variables used in this study along with the positive number of cases in different categories

\begin{tabular}{|l|l|l|l|l|l|l|l|}
\hline \multicolumn{2}{|l|}{ Characteristics } & $\begin{array}{l}\text { Number } \\
\text { of cases }\end{array}$ & $\begin{array}{l}\text { PD-1 } \\
\text { positive }\end{array}$ & $\begin{array}{l}\text { PD-L1 } \\
\text { positive TILs } \\
\text { (1\% cut-off) }\end{array}$ & $\begin{array}{l}\text { PD-L1 positive } \\
\text { (tm 1\% cut-off) }\end{array}$ & $\begin{array}{l}\text { PD-L1 positive } \\
\text { (TILs 5\% cut-off) }\end{array}$ & $\begin{array}{l}\text { PD-L1 positive } \\
\text { (tm 5\% cut-off) }\end{array}$ \\
\hline \multirow{4}{*}{ Age (y) } & $0-40$ & 13 & 4 & 10 & 8 & 9 & 6 \\
\cline { 2 - 8 } & $>40-60$ & 53 & 16 & 39 & 32 & 37 & 25 \\
\cline { 2 - 8 } & $>60$ & 50 & 18 & 43 & 32 & 33 & 28 \\
\hline \multirow{3}{*}{ Gender } & Male & 106 & 35 & 83 & 64 & 71 & 53 \\
\cline { 2 - 8 } & Female & 10 & 3 & 9 & 8 & 8 & 6 \\
\hline \multirow{2}{*}{$\begin{array}{l}\text { Muscle } \\
\text { invasion }\end{array}$} & Present & 43 & 21 & 37 & 33 & 30 & 29 \\
\cline { 2 - 8 } & Absent & 63 & 14 & 46 & 32 & 41 & 24 \\
\hline \multirow{2}{*}{ invasion } & Present & 95 & 35 & 81 & 64 & 70 & 53 \\
\cline { 2 - 8 } & Absent & 21 & 3 & 11 & 8 & 9 & 62 \\
\hline \multirow{3}{*}{ Grade } & High & 77 & 33 & 68 & 59 & 59 & 7 \\
\cline { 2 - 8 } & Low & 39 & 5 & 24 & 13 & 20 & 22 \\
\hline \multirow{2}{*}{ Smoking } & Present & 44 & 14 & 37 & 23 & 30 & 22 \\
\cline { 2 - 8 } & Absent & 46 & 18 & 36 & 30 & 31 & 52 \\
\hline
\end{tabular}

Abbreviations: TIL, tumor infiltrating lymphocytes; tm, tumor.

$(p=0.001)$ and tumor involving deep muscle $(p=0.006)$, while it showed a non-significant difference in the expression in lamina invasive and noninvasive tumors $(p=0.07)$. PD-L1 immunoexpression in tumor cells shows a higher expression in tumors with higher grade, lamina invasion, and deep muscle invasion; however, PD-L1 immunoexpression in TILs showed higher expression in higher grade and lamina invasive tumor but no difference in tumors involving deep muscle (for $p$-values, refer to - Table 2).

\section{Discussion and Conclusion}

Although five immunotherapeutic agents (PD-1/PD-L1 inhibitors) have been approved by the US-FDA for use in bladder tumors, the appropriate assay for patient selection has remained controversial to date. Immunohistochemistry for PD-L1 and PD-1 is the most commonly used assay; however, the guidelines for positivity and clones to be used remain vague. A recent guideline by CAP-ACP allows the use of laboratory-developed tests (LDTs) if properly validated clinically and in the laboratory to be used for the specific purpose for which it has been validated. ${ }^{3}$ In our study, we have tried to evaluate the expression of PD-1 and PD-L1 in urothelial carcinoma using clones NAT105 and CAL10, respectively, and compare it with previous studies. We also plan to compare the immunoexpression of these antibody clones with already established assays in further studies. Although these clones are evaluated in other tumors such as lung, breast, and renal cell carcinomas, the literature regarding its evaluation in bladder carcinoma is limited. ${ }^{4-7}$

PD-1 immunohistochemistry in the present study showed positivity in $32.7 \%$ of cases with a high expression in highgrade and muscle-invasive carcinomas. A high expression in high-grade and muscle-invasive carcinomas has also been shown in the previous study by Kawahara et al. ${ }^{8}$ Although previous studies have shown a slightly higher expression, this may be due to different clones used, a higher number of invasive and high-grade carcinomas, tumor heterogeneity, and the different cut-offs for positivity. ${ }^{9,10}$ We also experienced marked difficulty in establishing the staining protocol and interpretation of PD-1 immunohistochemistry. We observed that the staining intensity is mild to moderate with

Table 2 p-Values of Fisher's Exact Test for clinicopathological variables

\begin{tabular}{|l|l|l|l|l|l|l|}
\hline Characteristics & PD-1 & $\begin{array}{l}\text { PD-L1 TILs } \\
(1 \% \text { cut-off })\end{array}$ & $\begin{array}{l}\text { PD-L1 tm } \\
(1 \% \text { cut-off })\end{array}$ & $\begin{array}{l}\text { PD-L1 TILs } \\
\text { (5\% cut-off) }\end{array}$ & $\begin{array}{l}\text { PD-L1 tm } \\
\text { (5\% cut-off) }\end{array}$ & $\boldsymbol{n}$ \\
\hline Age & 0.885 & 0.292 & 0.962 & 0.959 & 0.627 & 116 \\
\hline Muscle Invasion & 0.006 & 0.150 & 0.008 & 0.677 & 0.005 & 106 \\
\hline Gender & 1 & 0.685 & 0.314 & 0.499 & 0.743 & 116 \\
\hline Grade & 0.001 & 0.001 & $<0.001$ & 0.011 & $<0.001$ & 116 \\
\hline Lamina invasion & 0.070 & 0.002 & 0.002 & 0.009 & 0.030 & 116 \\
\hline Smoking & 0.514 & 0.593 & 0.284 & 1 & 1 & 90 \\
\hline
\end{tabular}


marked tumor heterogeneity in the expression, which has been described in previous studies. ${ }^{11}$

PD-L1 expression in tumor cells was seen in $62 \%$ of cases when a cut-off of $1 \%$ was taken and $51 \%$ of cases when a cutoff of $5 \%$ was taken for positivity. Similarly, PD-L1 expression in tumor-infiltrating lymphocytes was seen in $79 \%$ of cases when a cut-off of $1 \%$ was taken as positivity and $68 \%$ of cases when a cut-off of $5 \%$ was taken for positivity. The clone used in this study showed a relatively higher expression as compared with that reported in previous studies; however, we cannot evaluate the optimal cut-off values and propose to do the same in further studies using established assays and follow-ups. ${ }^{12-15}$ PD-L1 expression at a cut-off of both $1 \%$ and $5 \%$ in TILs was higher in high-grade and lamina invasive tumors $(p<0.05)$ and there was no statistical difference in the expression in muscle-invasive or noninvasive tumors. PD-L1 expression in tumor cells showed a high expression in high-grade, lamina invasive, and also muscle-invasive tumors $(p<0.05)$. The correlation of PD-L1 expression with grade and muscle invasion has also been shown previously by Kawahara et al and other authors. ${ }^{8,16}$

Differences in age, gender, or smoking history did not show any significant difference in the expression of either PD-1 or PD-L1. A previous study by Holland et al correlating clinicopathological features with PD-1 and PD-L1 expression has also shown no impact of age and sex on the expression. ${ }^{17}$

Higher PD-L1 expression is related to a better overall response to treatment and survival as in multiple studies done previously. ${ }^{16,18-20}$ Recently, the US FDA has approved the use of Atezolizumab and Pembrolizumab for first-line use in platinum therapy-ineligible patients only in PD-L1positive tumors. ${ }^{21}$ This has opened up the scope of compulsory PD-L1 testing in patients with urothelial carcinoma ineligible for platinum-based therapies; however, the availability of companion diagnostic approved is scarce and laboratory dependent. There is a marked demand for the development and validation of laboratory-dependent tests so that these may be used before treatment. This will promote the use of immunotherapeutic agents on a larger scale even in resource-poor settings.

Our current study is limited by the absence of correlation with a validated diagnostic assay. Moreover, we have not ascertained a fixed cut-off criterion for PD-L1 but this is important for further studies so that the data can be correlated. We also tried to follow up the patients; however, only 8 out of the total patients can be followed up and because these patients belonged to different categories, a valid statistical study cannot be done. This is the first study of immunoexpression of both PD-1 and PD-L1 in the literature in bladder tumors in the Indian subcontinent to the best of our knowledge and can be used as a baseline study.

Based on the present study we conclude that PD-L1 clone CAL10 showed excellent expression and appears to be comparable to other clones; however, this needs to be further confirmed using validated assays. The high prevalence of PD1 and PD-L1 positivity irrespective of age and sex also points to the possible use of immunotherapeutic agents in all sex and age groups.

\section{Authors' Contributions}

Utpal Kumar contributed to the concept, data collection, literature search, Immunohistochemistry, primary manuscript, and editing of the final manuscript. Michael Leonard Anthony contributed to the concept, data collection, and editing of the final manuscript. Rishabh Sahai contributed to the concept, data collection, literature search, immunohistochemistry, and editing of the final manuscript. Ankur Mittal contributed to the concept, data collection, and editing of the final manuscript. Prashant Durgapal contributed to the concept, primary reporting, primary manuscript, and editing of the final manuscript. Sanjeev Kishore contributed to the concept, primary reporting, scholarly inputs. and editing of the final manuscript.

\section{Institutional Ethics Committee Approval}

The study was approved by the Institution's Ethics Committee (approval no: AIIMS/IEC/20/185).

\section{Conflict of Interest}

None declared.

\section{Acknowledgments}

I thank Mr. Chandan and Mr. Tarakeshwar for extending their technical support.

\section{References}

1 Stenehjem DD, Tran D, Nkrumah MA, Gupta S. PD1/PDL1 inhibitors for the treatment of advanced urothelial bladder cancer. OncoTargets Ther 2018;11:5973-5989

2 U.S. Food \& Drug. List of cleared or approved companion diagnostic devices (in vitro and imaging tools) | FDA [Internet]. [cited 2020 Jun 2]. Accessed September 23, 2021 at: https://www.fda. gov/medical-devices/vitro-diagnostics/list-cleared-or-approvedcompanion-diagnostic-devices-vitro-and-imaging-tools

3 Cheung CC, Barnes P, Bigras G, et al; Canadian Association of Pathologists-Association Canadienne Des Pathologistes' National Standards Committee for High Complexity Testing (CAP-ACP NSCHCT) Fit-for-purpose PD-L1 biomarker testing for patient selection in immuno-oncology: guidelines for clinical laboratories from the Canadian Association of Pathologists-Association Canadienne Des Pathologistes (CAP-ACP). Appl Immunohistochem Mol Morphol 2019;27(10):699-714

4 Karnik T, Kimler BF, Fan F, Tawfik O. PD-L1 in breast cancer: comparative analysis of 3 different antibodies. Hum Pathol 2018; 72:28-34

5 Zarogoulidis P, Porpodis K, Xatzibougias D, Tsiouda T, Daniilidis D. PD-L1 evaluation with DAKO and BIOCARE antibody for immunotherapy; same coin different faces. Eur Respir J 2019;54:PA3072. Doi: 10.1183/13993003.congress-2019.PA3072

6 Kang MJ, Kim KM, Bae JS, et al. Tumor-infiltrating PD1-positive lymphocytes and FoxP3-positive regulatory T cells predict distant metastatic relapse and survival of clear cell renal cell carcinoma. Transl Oncol 2013;6(03):282-289

7 Tumeh PC, Harview CL, Yearley JH, et al. PD-1 blockade induces responses by inhibiting adaptive immune resistance. Nature 2014;515(7528):568-571

8 Kawahara T, Ishiguro Y, Ohtake S, et al. PD-1 and PD-L1 are more highly expressed in high-grade bladder cancer than in low-grade cases: PD-L1 might function as a mediator of stage progression in bladder cancer. BMC Urol 2018;18(01):97 
9 Xylinas E, Robinson BD, Kluth LA, et al. Association of T-cell coregulatory protein expression with clinical outcomes following radical cystectomy for urothelial carcinoma of the bladder. Eur J Surg Oncol 2014;40(01):121-127

10 Le Goux C, Damotte D, Vacher S, et al. Correlation between messenger RNA expression and protein expression of immune checkpoint-associated molecules in bladder urothelial carcinoma: a retrospective study. Urol Oncol 2017;35(05):257-263

11 Owen D, Chu B, Lehman AM, et al. Expression patterns, prognostic value, and intratumoral heterogeneity of PD-L1 and PD-1 in thymoma and thymic carcinoma. J Thorac Oncol 2018;13(08): 1204-1212

12 Wang B, Pan W, Yang M, et al. Programmed death ligand-1 is associated with tumor infiltrating lymphocytes and poorer survival in urothelial cell carcinoma of the bladder. Cancer Sci 2019; 110(02):489-498

13 Pichler R, Fritz J, Lackner F, et al. Prognostic value of testing PD-L1 expression after radical cystectomy in high-risk patients. Clin Genitourin Cancer 2018;16(05):e1015-e1024

14 Li Q Li F, Che J, Zhao Y, Qiao C. Expression of B7 homolog 1 (B7H1) is associated with clinicopathologic features in urothelial bladder cancer. Med Sci Monit 2018;24:7303-7308

15 Wang Y, Zhuang Q, Zhou S, Hu Z, Lan R. Costimulatory molecule B7-H1 on the immune escape of bladder cancer and its clinical significance. J Huazhong Univ Sci Technolog Med Sci 2009;29(01): 77-79
16 Ding X, Chen Q Yang Z, et al. Clinicopathological and prognostic value of PD-L1 in urothelial carcinoma: a meta-analysis. Cancer Manag Res 2019;11:4171-4184

17 Holland BC, Sood A, Delfino K, et al. Age and sex have no impact on expression levels of markers of immune cell infiltration and immune checkpoint pathways in patients with muscle-invasive urothelial carcinoma of the bladder treated with radical cystectomy. Cancer Immunol Immunother 2019;68(06):991-997

18 Zajac M, Ye J, Mukhopadhyay P, et al. Optimal PD-L1-high cutoff for association with overall survival in patients with urothelial cancer treated with durvalumab monotherapy. PLoS One 2020;15 (04):e0231936

19 Plimack ER, Bellmunt J, Gupta S, et al. Pembrolizumab (MK-3475) for advanced urothelial cancer: Updated results and biomarker analysis from KEYNOTE-012. J Clin Oncol 2015;33(15_suppl): 4502-4502

20 Massard C, Gordon MS, Sharma S, et al. Safety and efficacy of durvalumab (MEDI4736), an anti-programmed cell death ligand-1 immune checkpoint inhibitor, in patients with advanced urothelial bladder cancer. J Clin Oncol 2016;34(26): 3119-3125

21 Eckstein M, Erben P, Kriegmair MC, et al. Performance of the Food and Drug Administration/EMA-approved programmed cell death ligand- 1 assays in urothelial carcinoma with emphasis on therapy stratification for first-line use of atezolizumab and pembrolizumab. Eur J Cancer 2019;106:234-243 